\title{
Beyond Cholinesterase Inhibition: Anti-inflammatory Role and Pharmacological Profile of Current Drug Therapy for Alzheimer's Disease
}

\author{
Franco Giubilei* \\ NESMOS Department - University of Roma "Sapienza” - Italy
}

\begin{abstract}
Inflammation is a common response of an individual against either exogenous or endogenous damage. The role of inflammation and of inflammatory cells recently emerged also in the pathogenesis of neurodegenerative disorders. Experimental evidences show how neurotransmitters, besides their role in the synapses, play a modulatory role during immune response. Drugs used for treatment of dementia symptoms are able to increase neurotransmitters levels, and likely to have a modulatory role during immune response. Aim of this review is to discuss the most recent advances on inflammation role during neurodegeneration and also to individuate the potential anti-inflammatory role played by drugs currently used for Alzheimer's disease treatment.
\end{abstract}

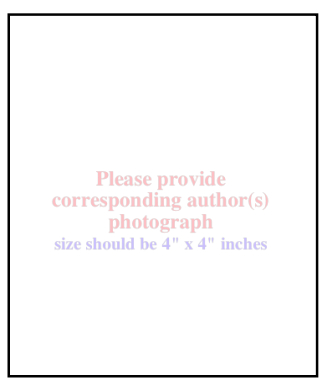

Keywords: Alzheimer's Disease, cholinesterase inhibitors, inflammation, neurodegeneration glia, nicotinic receptor.

Received: October 31, 2015

Revised: December 11, 2015

Accepted: December 15, 2015

\section{INTRODUCTION}

Inflammatory response is essential for the survival of an organism - it consists of a mechanism of self-defense triggered by the immune system against endogenous and exogenous damage. Inflammation must be closely regulated because an insufficient or an excessive response will lead to pathological conditions, such as immunodeficiency or chronic inflammatory diseases [1]. The role of inflammation in some acute brain pathologies, such as multiple sclerosis, encephalitis and acute neurodegeneration following ischemia or trauma, has been widely described [2]. By contrast, its role in some neurodegenerative diseases, such as Alzheimer's disease (AD), is not clear.

The immune system is negatively influenced by aging. Normal human aging, which is the consequence of a dysfunction of self-organizing systems and of their reduced ability to adapt to the environment, has been associated with a loss of complexity in many anatomical structures and physiological processes [3]. Progressive changes in the immune system over a person's lifespan have been reported to influence the capacity to respond to immune challenges. Both T-cell and B-cell systems are believed to be involved in this process [4-7]. These age-associated immune changes are called immunosenescence [8]. It has been hypothesized that human immunosenescence is characterized by a deterioration in adaptive immunity, whereas innate immunity is conserved or even up-regulated. These changes in the immune system may result from continual exposure to a range of potential

\footnotetext{
*Address correspondence to this author at the NESMOS Department Università Sapienza di Roma, II Facoltà di Medicina e Chirurgia; Azienda Ospedaliera Sant'Andrea, Via Grottarossa, 1035-00189 Roma - Italy; Tel: $003906 \quad 33775525$; Fax: $003906 \quad 33775900$; E-mail: franco.giubilei@uniroma1.it
}

antigens, such as viruses, bacteria, food and self-molecules $[9,10]$. Within this scenario, immunosenescence is believed to reduce the ability of older people to control infectious diseases. Furthermore, it has been hypothesized that the immune system is implicated, to a varying extent, in many age-related diseases. Indeed, the prevalence of cancer and chronic inflammatory and autoimmune diseases, as well as that of neurodegenerative diseases, is the highest in this phase of life [11]. By contrast, a good immune system in older people has been correlated with health status. One possible explanation for these findings may lie in the increased serum levels of inflammatory mediators, such as cytokines and acute phase proteins, observed in older people, which is indicative of a chronic low-grade inflammatory state [12]. The prolonged duration of this inflammatory state may damage several organs, including the brain. Chronic inflammation thus appears to be involved in the pathogenesis of all age-related diseases, including atherosclerosis, diabetes, heart disease and cancer [13, 14]. A growing body of evidence also suggests that inflammation plays an important role in various neurodegenerative diseases, to which $\mathrm{AD}$ has more recently been added $[2,15]$.

Acetylcholinesterase inhibitors (AChEIs) are widely used for the symptomatic treatment of AD. Although the primary action of all currently available AChEIs is based on their ability to inhibit acetylcholinesterase (AChE), the evidence suggests that they possess other mechanisms, such as an antiinflammatory effect, that may be exploited to treat AD. If so, AChEIs may to some extent be able to modify the disease course. The aim of this review is to provide an overview of the anti-inflammatory mechanism of AChEIs and a summary of their pharmacological profiles.

INFLAMMATION IN ALZHEIMER'S DISEASE 
Although there is emerging evidence that the causes of $\mathrm{AD}$ often overlap the other forms of dementia, AD remains the most common cause of dementia, accounting for $60-80 \%$ of cases. It is estimated that approximately 34 million people worldwide have AD [16]. The two main neuropathological hallmarks of $\mathrm{AD}$ are extracellular amyloid-beta $(\mathrm{A} \beta)$ plaques and intracellular neurofibrillary tangles accompanied by reactive microgliosis, dystrophic neuritis and loss of neurons and synapses [17]. According to the $A \beta$ hypothesis, the aggregation of $A \beta$ has been identified as the crucial factor in the development of the disease, whereas brain inflammation is considered to be a response aimed at eliminating the initial cause of cell injury as well as necrotic cells and tissue. Although the underlying causes of these changes remain unknown, aging and genetic factors are believed to play the most important role. Inflammation has consequently long been considered to be a pathological hallmark of $\mathrm{AD}$, though not to play an active role in the pathogenesis of the disease. However, emerging evidence suggests that inflammation does instead play an active role in the pathogenesis of this disease through certain reactions, such as those associated with $A \beta$ plaques and neurofibrillary tangles [18].

The cells responsible for this inflammatory reaction are microglia and astrocytes [15]. The main role of microglia is to surveil brain tissue for the presence of pathogens and cellular debris as well as to maintain the plasticity of neuronal circuits [19]. In normal healthy brain, microglial cells display a typical down-regulated phenotype when compared with other tissue macrophages, but rapidly react in response to a number of acute and chronic insults. Activated microglial cells may cause neuronal damage by releasing free radicals as well as cytokines and toxic factors. Alternatively, microglia may exert neuroprotective functions by secreting growth factors or diffusible anti-inflammatory mediators, which help to resolve inflammation and restore tissue homeostasis [20]. In $\mathrm{AD}$ brain, microglia link $\mathrm{A} \beta$ via cell-surface receptors, thereby leading to the production of pro-inflammatory cytokines and chemokines [21-23]. At the same time, microglia start to engulf $A \beta$ fibrils by phagocytosis in response to receptor ligation. These mechanisms ultimately lead to the activation of a chronic inflammatory reaction [23]. Inflammatory mediators, in turn, increase the production of the $\mathrm{A} \beta 42$ peptide, which induces the expression of pro-inflammatory cytokines in glial cells in a vicious cycle [24]. These results support the role of microglia in the chronic inflammation present in most neurodegenerative diseases, including $\mathrm{AD}$. In these pathophysiological conditions, microglia may remain activated for many years, during which they secrete various inflammatory factors. The contribution of blood-derived mononuclear cells that infiltrate the central nervous system (CNS) is also unclear within this scenario. There is, however, evidence pointing to an involvement of the systemic immune response in the pathogenesis of AD. In particular, animal studies have shown infiltration of bloodderived cells in AD brain, while other studies have suggested that peripheral mononuclear phagocytes reduce the development of $A \beta$ plaques [25]. There is also evidence indicating that peripheral blood mononuclear cells exert their effect without entering the CNS. In particular, peripheral blood mononuclear cells in $\mathrm{AD}$ patients produce higher levels of pro-inflammatory cytokines than those in normal subjects [26]. Furthermore, reports of changes in lymphocyte distribution and in cytokine levels in the plasma of $\mathrm{AD}$ patients point to the involvement of the immune system in $\mathrm{AD}[27]$.

All these findings suggest that it may be possible to treat AD by means of anti-inflammatory drugs. This pharmacological approach is also supported by the results of epidemiological studies showing that non-steroid antiinflammatory drug (NSAID) treatment confers protective effects associated with a reduced incidence of $\operatorname{AD}[28,29]$. Anti-inflammatory randomized controlled trials have, however, yielded negative results. The drugs tested include prednisone, hydroxychloroquine, indomethacin, tarenflurbil and inhibitors of COX-1 and COX-2 [30-35].

\section{INFLAMMATION AND THE CHOLINERGIC SYSTEM}

One important endogenous mechanism that regulates the inflammatory response is a cross-talk between the immune and nervous systems. Numerous reports suggest that acetylcholine (ACh) might function as an important modulator of cellular interactions and immune functions [36, 37]. In particular, electric stimulation of the vagus nerve has been shown to attenuate inflammation during endotoxemia in rats [38]. Moreover, $\mathrm{ACh}$, the main vagal neurotransmitter, deactivates peripheral macrophages and inhibits the release of pro-inflammatory mediators. In human macrophage cultures, ACh significantly reduces the release of proinflammatory cytokines, such as interleukin (IL)-1 $\beta$, IL-6, IL-18 and tumor necrosis factor (TNF), without affecting the release of IL-10, an anti-inflammatory cytokine [38]. In the peripheral nervous system (PNS), acetylcholine-dependent macrophage deactivation is mediated by the $\alpha 7 \mathrm{r}$ subunit of the nicotinic acetylcholine receptor (nAChR), which is expressed in peripheral macrophages and has been described as being indispensable to the so-called "cholinergic antiinflammatory pathway" [39]. A brain cholinergic pathway that regulates microglia activation through $\alpha 7 \mathrm{nAChRs}$ has consequently been hypothesized [40, 41]. In addition to neurons and peripheral macrophages, several studies have demonstrated the expression of nAChRs in cell types both within and outside the nervous system [42]. Cultured hippocampal astrocytes express functional $\alpha 7$ receptors [43] while cortical astrocytes express both nicotinic and muscarinic receptors [44]. These data suggest that ACh may have an active role in attenuating inflammation in both brain and periphery.

\section{ACETYLCHOLINESTERASE INHIBITORS}

The pathogenesis of AD has for many years been linked to a deficit of the neurotransmitter Ach in the brain. This hypothesis is supported by evidence indicating that cognitive impairment is correlated with cholinergic system dysfunctions [45]. A reduction in a number of cholinergic markers, such as choline acetyltransferase, ACh receptor bindings and ACh concentrations in the synaptic space, has been reported [46]. Over the years, it has been emerged that cholinergic dysfunction is not a primary neuropathological 
hallmark of disease but rather a consequence of brain degeneration. Nevertheless, clinical trials based on a strategy aimed at correcting the ACh system dysfunction led to the first licensed medication for AD. Three AChEIs are currently being used to treat patients affected by AD: donepezil, galantamine and rivastigmine. All three AChEIs are licensed for the treatment of mild to moderate $\mathrm{AD}$, though donepezil is also approved for severe AD in the USA. Rivastigmine is also approved for the treatment of dementia associated with Parkinson's disease by North America and European regulatory agencies. Tacrine, the first AChEI on the market, has been replaced with other AChEIs owing to its serious adverse effects [47]. Another AChEI proposed is huperzine A, which has been approved for the treatment of mild to moderate AD in China, though not in North America or in European Countries. Huperzine A has displayed some benefits on dementia symptoms, though the results are inconclusive because of weaknesses in the methodology adopted for the clinical trials [48]. A phase II clinical trial yielded a significant effect on cognition though not on function or global status [49].

The use of AChEIs has been shown to have a significant, albeit modest, therapeutic effect on dementia symptoms, including activities of daily living, with no significant difference emerging between the three AChEIs [50, 51]. Furthermore, only $15-35 \%$ of all $\mathrm{AD}$ patients treated with AChEIs respond to these drugs. Several reasons have been suggested to explain the inter-individual variability of the drug response to AChEIs, such as co-morbidities, drug interactions, compliance, adverse reactions, genetic variations [52]. The mechanism of action underlying AChEIs is the inhibition of $\mathrm{AChE}$, which reduces the breakdown of $\mathrm{ACh}$ in the synaptic cleft. Two forms of cholinesterase are present in humans, i.e. butyrylcholinesterase (BuChE) and AChE, which are predominant in the PNS and CNS, respectively [53]. While the physiological function of $\mathrm{BuChE}$ is not yet clear, $\mathrm{AChE}$ concludes the action of $\mathrm{ACh}$ by hydrolyzing it to choline and acetate [54]. The AChE in the brain has two forms, i.e. monomeric G1 and tetrameric G4. In AD brain it is only the latter form that displays a selective reduction of its activity [55].

Very few studies have been planned to investigate the anti-inflammatory capacity of AChEIs in patients affected by AD. Richardson and colleagues [56] examined the long-term use of AChEIs on pro-inflammatory cytokines in the periphery in $A D$ patients. They did not find any difference between patients treated with AChEIs and drug naïve patients in relation to the concentrations of IL-1 $\beta$, IL- 6 and TNF- $\alpha$. Furthermore, the levels of cytokines did not differ among the three AChEIs: donepezil, galantamine and rivastigmine. Some limitations of the study such as the lack of longitudinal follow-up and the small sample may have influenced these results that are in contrast to animal studies that suggest an anti-inflammatory role for AChEIs. Therefore, a more detailed pharmacological profile of each AChEI may help to better understand their potential antiinflammatory action.

\section{Donepezil}

Donepezil binds to AChE in a reversible and noncompetitive manner, without involving BuChE inhibition.
Due to its long plasma half-life, approximately 70 hours, donepezil can be administered once daily [57]. Immediaterelease tablets $(5 \mathrm{mg}$ and $10 \mathrm{mg}$ ) and a $23 \mathrm{mg}$ sustainedrelease tablets are available. Food intake doesn't significantly influence the drug absorption. Donepezil is metabolized by the enzymes cytochrome P450 (CYP) 3A4 and 2D6 in the liver, and the elimination of the parent drug and the metabolites is renal [58]. Clinical monitoring is therefore recommended when donepezil is administered together to other CYP3A4 and CYP2D6 inhibitors. Although some pharmacokinetic parameters are affected by aging, modification of donepezil dose is not necessary in the elderly [59]. Randomized clinical trial found significant beneficial effects on cognition, function, and global status for $\mathrm{AD}$ patients receiving donepezil $5 \mathrm{mg} /$ day and $10 \mathrm{mg} /$ day for 3-6 months $[50,60-62]$. The effect of donepezil on neuropsychiatric symptoms is rather controversial. Some meta-analysis showed a significant positive effect on behavioral in $\mathrm{AD}$ patients treated with donepezil $[50,60]$. However, studies focused on the neuropsychiatric symptom treatment of $\mathrm{AD}$ patients did not find any significant difference between donepezil and placebo [63, 64]. Significant improvement in cognition in a dose-dependent manner was observed in a meta-analysis including $3320 \mathrm{AD}$ patients treated with donepezil [62]. In this analysis a beneficial effect on global status, independent of dose, was also found. A 24-week, randomized, double-blind study found that donepezil $23 \mathrm{mg}$ /day treatment provided a small but significant improvement in the cognition endpoint compared with donepezil $10 \mathrm{mg} /$ day treatment in moderate to severe $\mathrm{AD}$ patients [65].

Recent experimental studies suggest that activated microglia may be one of the direct targets of donepezil in the central nervous system. Using purified microglia cultures and microglia cell lines, Hwang and colleagues [66] found that donepezil attenuated microglia production of nitric oxide and TNF- $\alpha$ and suppressed the gene expression of nitric oxide, IL-1 $\beta$ and TNF- $\alpha$. In microglia/neuroblastoma coculture and animal model of neuroinflammation, they furthermore described the inhibitory effects of donepezil on microglial activation. It should be noted, however, that the concentration of donepezil used in these clinical experiments is much higher than that used clinically. These results were confirmed by Kim and colleagues [67] that examined the anti-inflammatory effect of donepezil against $A \beta$-oligomers and its neuroinflammatory mechanisms in cultured microglial cells and in mice. They found that donepezil significantly attenuated the release of inflammatory mediators from microglia and that it suppressed activated microglia-mediated toxicity in primary hippocampal cells. Further experimental studies have showed that donepezil was able to prevent pro-inflammatory cytokines, lipid peroxidation and memory impairments induced in mice brain by various substances [68-70]. Finally, eight months of donepezil treatment resulted in improvement of tau pathology, synaptic and neuronal loss as well as of neuroinflammation [70]. Interesting results have been found investigating peripheral blood mononuclear cells of patients affected by AD. Compared with untreated patients and healthy subjects, IL1 $\beta$ levels and expression were decreased as well as IL-4 levels and expression were significantly 
higher in AD patients treated with donepezil, suggesting an active role of the drug in reducing peripheral inflammatory markers $[71,72]$.

\section{Galantamine}

Galantamine is a competitive inhibitor of $\mathrm{AChE}$ and an allosteric modulator of nAChR [73]. It has a linear pharmacokinetics and approximately 7 hours plasma half-life [74]. In addition to a twice-daily immediate-release preparation, an extended-release capsule formulation of galantamine was developed, allowing once-daily administration [75]. Galantamine is metabolized by the CYP $3 \mathrm{~A} 4$ and 2D6, and the elimination of the parent drug and the metabolites is renal. About $30 \%$ of galantamine is excreted unchanged in the urine [76]. Galantamine clearance is decreased with aging [77]. When galantamine is administered together to other CYP3A4 and CYP2D6 inhibitors, dose reduction may be required. Evidence suggests caution in the use of galantamine in patients with moderate impairment of hepatic function, and galantamine is not recommended in severe hepatic dysfunction [78].

Because of its capacity to modulate of nAChRs for improving nicotinic transmission, galantamine might interact with the cholinergic anti-inflammatory pathway [79]. At this regard, Pavlov and colleagues [80] reported evidence suggesting that the $\alpha 7 \mathrm{nAChR}$-mediated cholinergic antiinflammatory pathway is required for the anti-inflammatory effect of galantamine. They hypothesized that inhibition of brain AChE suppress systemic inflammation through a central muscarinic receptor-mediated and vagal- and

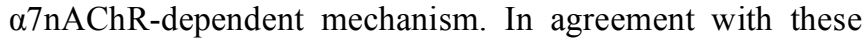
data, Liu $\mathrm{ZH}$ and colleagues [81] found that galantamine treatment $(3 \mathrm{mg} / \mathrm{Kg})$ reduced the level of circulating TNF- $\alpha$ in rats with lipopolysaccharide-induced peritonitis. At the same time, they reported evidence that the vagus nerve plays a role in the process of the action of galantamine. Further experimental studies described the role of neural cholinergic signaling in controlling inflammation and demonstrated that galantamine can reduce excessive pro-inflammatory cytochine release $[80,82,83]$. Finally, Takata and colleagues [84] showed that galantamine treatment facilitated $A \beta$ clearance in brains of rodent AD models. They suggested that galantamine sensitizes microglial $\alpha 7 \mathrm{nAChRs}$ to choline and induces calcium influx into microglia which in turn, may stimulate $\mathrm{A} \beta$ phagocytosis.

\section{Rivastigmine}

Rivastigmine is an inhibitor of both $\mathrm{AChE}$ and BChE. It easily crosses the blood brain barrier and inhibits the AChE in a pseudo-irreversible way, due to its persistent action [85]. The inactivation of AChE enzyme last more than 24 hours, because of the carbamyl moiety of rivastigmine that remains bound to its substrate [86]. Rivastigmine has a very short plasma half-life of 1,5-2 hours. Since gastrointestinal adverse effects have been associated to the high plasma concentration values, it is recommended to administer rivastigmine with food. To improve the tolerability of rivastigmine, a transdermal path has been developed. This formulation gradually releases the drug over the 24 hours, avoiding significantly the wide fluctuation in the plasma concentration
[87]. Rivastigmine is not significantly metabolized by hepatic microsomal enzymes, making drug-drug interactions unlikely [88]. Pharmacokinetic parameters are little influenced by aging but a dose titration of the drug must be performed according to its tolerability. A meta-analysis conducted by Cochrane collaboration found that only high dose of rivastigmine (6-12 $\mathrm{mg}$ daily) showed significant beneficial effects on cognition, function and global status [89]. Using low dose treatment (1-4 mg daily), a significant beneficial effect was only found on cognitive functions. No significant difference was found between rivastigmine and placebo in behavioral disturbances. In IDEAL study the effects of small transdermal rivastigmine patch $(9.5 \mathrm{mg}$ daily), large transdermal rivastigmine patch (17.4 mg daily) and oral rivastigmine capsules (6-12 $\mathrm{mg}$ daily) were compared [90]. All rivastigmine treatment groups showed significant improvement relative to placebo on cognition, behavior and global impression of change. The small patch had similar efficacy to the capsule, with approximately twothirds fewer reports of nausea and vomiting as well as no significant differences between the small transdermal rivastigmine patch and the large one were found. Other meta-analysis studies confirmed that rivastigmine has significant beneficial effects on cognition, function and global status $[60,62]$. However, in a meta-analysis study, Raina and colleagues [50] reported that rivastigmine produces significant improvement only on global status in $\mathrm{AD}$ patients while the results regarding cognitive outcomes and functional abilities were inconsistent.

In experimental autoimmune encephalomyelitis, rivastigmine reduced demyelination, microglia activation and axonal damage as well as decreased the production of pro-inflammatory cytokines (TNF- $\alpha$, Interferon- $\gamma$ and IL17), without affecting IL-10 production [91]. These effects were abolished by $\alpha 7 \mathrm{nAChR}$ antagonists, suggesting the implication of the cholinergic anti-inflammatory pathway in the process. The same conclusions were reported by Shifrin [92] that investigated the capacity of rivastigmine to improve the pathology of colitis in mice and rats by increasing the concentration of $\mathrm{ACh}$ in the brain and periphery. Rivastigmine significantly decreased the release of nitric oxide, TNF- $\alpha$, IL- $1 \beta$ and IL- 6 and this effect was abolished by $\alpha 7$ nicotinic receptor blockade. In AD patients treated with rivastigmine, IL-6 level was $47 \%$ lower than the average value of the AD patients treated with other drugs [93].

\section{CONCLUSION}

Emerging evidence suggests that inflammation plays an active role in the pathogenesis of $\mathrm{AD}$ through certain reactions, such as those associated with $A \beta$ plaques and neurofibrillary tangles and that $\mathrm{ACh}$ may have an active role in attenuating inflammation in both brain and periphery. A link between inflammation and cholinergic system has been identified in the anti-inflammatory role of vagus nerve. Experimental and animal studies showed that AChEIs inhibit the release of cytokines from microglia and monocytes. Surprisingly, there are very few researchers that investigated the effects of AChEIs on peripheral inflammatory cytokines in subjects affected by AD. The symptomatic efficacy of AChEIs is due to their augmentation of Ach-mediated 
neuron-to-neuron transmission. However, we can hypothesize that the efficacy of AChEIs is, at least in part, due to an anti-inflammatory action. More research is now needed to clarify the anti-inflammatory role of AChEIs in AD patients and to define the mechanisms involved.

\section{LIST OF ABBREVIATIONS:}

\begin{tabular}{|c|c|c|}
\hline Ach & $=$ & AcetylCholine \\
\hline AChEI & $=$ & AcetylCholine Esterase Inhibitors \\
\hline $\mathrm{AD}$ & $=$ & Alzheimer's Disease \\
\hline $\mathrm{A} \beta$ & $=$ & Amyloid Beta \\
\hline $\mathrm{BuChE}$ & $=$ & Butyrylcholine Esterase \\
\hline CNS & $=$ & Central Nervous System \\
\hline IL & $=$ & Interleukine \\
\hline NAChR & $=$ & nicotinic Acetyl Choline Receptor \\
\hline NSAID & $=$ & Nonsteroidal Antinflammatory Drugs \\
\hline PNS & $=$ & Peripheral Nervous System \\
\hline TNF & $=$ & Tumor Necrosis Factor \\
\hline
\end{tabular}

\section{CONFLICT OF INTEREST}

The author(s) confirm that this article content has no conflict of interest.

\section{ACKNOWLEDGEMENTS}

Declared none.

\section{REFERENCES}

[1] Nathan C. Points of control in inflammation. Nature 2002; 420: 846-52.

[2] Perry VH, Newman TA, Cunningham C. The impact of systemic infection on the progression of neurodegenerative disease. Nat Rev Neurosci 2003; 4(2): 103-12.

[3] Goldberger AL, Peng CK, Lipsitz LA. What is physiologic complexity and how does it change with aging and disease? Neurobiol Aging 2002; 23(1): 23-6.

[4] Pawelec G and Larbi A. Immunity and ageing in man: annual review 2006/2007. Exp Gerontol 2008; 43: 34-8.

[5] Sauce D and Appay V. Altered thymic activityin early life: how does it affect the immune system in young adults? Curr Opin Immunol 2011; 23: 543-8.

[6] Pawelec G, McElhaney JE, Aiello AE, Derhovanessian E. The impact of CMV infection on survival in older humans. Curr Opin Immunol 2012; 24(4): 507-11.

[7] Buffa S, Bulati M, Pellicanò M, et al. B cell immunosenescence: different features of naive and memory B cells in elderly. Biogerontology 2011; 12(5): 473-83.

[8] Martorana A, Bulati M, Buffa S, et al. Immunosenescence, inflammation and Alzheimer's disease. Longev Healthspan 2012; 1: 8 .

[9] Akbar AN, Henson SM. Are senescence and exhaustion intertwined or unrelated processes that compromise immunity? Nat Rev Immunol 2011; 11: 289-95.

[10] Henson SM, Riddell NE, Akbar AN. Properties of end-stage human $\mathrm{T}$ cells defined by CD45RA re-expression. Curr Opin Immunol 2012; 24(4): 476-81.

[11] Vasto S, Caruso C. Immunity \& Ageing: a new journal looking at ageing from an immunological point of view. Immun Ageing 2004; 1: $1-4$.
[12] Krabbe KS, Pedersen M, Bruunsgaard H. Inflammatory mediators in the elderly. Exp Gerontol 2004; 39(5): 687-99.

[13] Spirig R, Tsui J, Shaw S. The Emerging Role of TLR and Innate Immunity in Cardiovascular Disease. Cardiol Res Pract 2012; 2012: 181394.

[14] Coussens LM, Werb Z. Inflammation and cancer. Nature 2002; 420: 860-7.

[15] Heneka MT, Carson MJ, El Khoury J, et al. Neuroinflammation in Alzheimer's disease. Lancet Neurol 2015; 14(4): 388-405.

[16] Brookmeyer R, Johnson E, Ziegler-Graham K, Arrighi HM. Forecasting the global burden of Alzheimer's disease. Alzheimers Dement 2007; 3(3): 186-91.

[17] Serrano-Pozo A, Frosch MP, Masliah E, Hyman BT. Neuropathological alterations in Alzheimer disease. Cold Spring Harb Perspect Med 2011; 1(1): a006189.

[18] Zhang B, Gaiteri C, Bodea LG, et al. Integrated systems approach identifies genetic nodes and networks in late-onset Alzheimer's Dis Cell 2013; 153(3): 707-20.

[19] Ji K, Miyauchi J, Tsirka SE. Microglia: an active player in the regulation of synaptic activity. Neural Plast 2013; 2013: 627325.

[20] Streit WJ. Microglia as neuroprotective, immunocompetent cells of the CNS. Glia 2002; 2: 133-9.

[21] Liu Y. et al. LPS receptor (CD14): a receptor for phagocytosis of Alzheimer's amyloid peptide. Brain 2005; 128: 1778-89.

[22] Querfurth HW, La Ferla FM. Alzheimer's disease. N Engl J Med 2010; 362: 329-44.

[23] Lee CYD, Landreth GE. The role of microglia in amyloid clearance from the AD brain. J Neurol Transm 2010; 117: 949-60.

[24] Linderberg C, et al. Cytokine production by a human microglial cell line: effects of $\beta$-amyloid anda-melanocyte-stimulating hormone. Neurotox Res 2005; 8: 267-76.

[25] Simard AR, et al. Bone marrow-derived microglia play a critical role in restricting senile plaque formation in Alzheimer's disease. Neuron 2006; 49: 489-502.

[26] Reale $\mathrm{M}$, et al. Acetylcholinesterase inhibitors effects on oncostatin-M, interleukin-1 beta and interleukin-6 release from lymphocytes of Alzheimer's disease patients. Exp Gerontol 2005; 40: 165-71.

[27] Pellicanò $\mathrm{M}$, et al. Immune profiling of Alzheimer patients. J Neuroimmunol 2012; 242: 52-59.

[28] McGeer PL, et al. Arthritis and anti-inflammatory agents as possible protective factors for Alzheimer's disease: a review of 17 epidemiologic studies. Neurology 1996; 47: 425-432

[29] Szekely CA, et al. Nonsteroidal anti-inflammatory drugs for the prevention of Alzheimer's disease: a systematic review. Neuroepidemiology 2004; 23: 1105-1113

[30] Van Gool WA, et al. Effect of hydroxychloroquine on progression of dementia in early Alzheimer's disease: an 18-month randomized, double-blin, placebo-controlled study. Lancet 2001; 358: 455-460.

[31] Reines SA, et al. Rofecoxib: no effect on Alzheimer's disease in a 1-year, randomized, blinded, controlled study. Neurology 2004; 62: 66-71

[32] Aisen PS, et al. Effects of rofecoxib or naproxen vs placebo on Alzheimer's disease progression: a randomized controlled trial. JAMA 2003; 289: 2819-2826

[33] Aisen PS, et al. A randomized controlled trial of prednisolone in Alzheimer's disease. Alzheimer's Disease Cooperative Study. Neurology 2000; 54: 455-460

[34] De Jong D, et al. No effect of one-year treatment with indomethacin on Alzheimer's disease progression: a randomized controlled trial. PLoS One 2008; 3: e1475

[35] Green RC, et al. Effect of tarenflurbil on cognitive decline and activities of daily living in patients with mild Alzheimer disease: a randomized controlled trial. JAMA 2009; 302: 2557-2564

[36] Wang Y, et al. Human bronchial epithelial and endothelial cells express alpha 7 nicotinic acetylcholine receptors. Mol Pharmacol 2001; 60: 1201-1209

[37] Matsunaga K, et al. Involvment of nicotinic acetylcholine receptors in suppression of antimicrobial activity and cytokine responses of alveolar macrophages to Legionella pneumophilainfection by nicotine. J Immunol 2001; 167: 6518-6524

[38] Borovikova LV, et al. Vagus nerve stimulation attenuates the sistemi inflammatory response to endotoxin. Nature 2000; 405: 458-462. 
[39] Wang $\mathrm{H}$, et al. Nicitinic acetylcholine receptor $\alpha 7$ subunit is an essential regulator of inflammation. Nature 2003; 421:384-388

[40] Shytle RD, et al. Cholinergic modulation of microglial activation by $\alpha 7$ nicotinic receptors. J Neurochem 2004; 89: 337-343

[41] De Simone R, et al. Activation of $\alpha 7$ nicotinic acetylcholine receptor by nicotine selectively up-regulates cyclooxygenase- 2 and prostaglandin E2 in rat microglia cultures. J Neuroinflamation 2005; $2: 4$

[42] Sharma G and Vijayaraghavan S. Nicotinic receptor signaling in non-excitable cells. J Neurobiol 2002; 53: 524-534

[43] Sharma G and Vijayaraghavan S. Nicotinic cholinergic signaling in hippocampal astrocytes involves calcium-induced calcium release from intracellular stores. Proc Natl Acad Sci USA 2001; 98: 41484153

[44] Hosli E, et al. Colocalization of androgen, estrogen and cholinergic receptors on cultured astrocytes of rat central nervous system. Int $\mathbf{J}$ Dev Neurosci 2001; 1: 11-19.

[45] Perry EK, et al. Correlation of cholinergic abnormalities with senile plaques and mental test scores in senile dementia. Br Med J 1978; 6150: 1457-1459

[46] Schliebs $\mathrm{R}$ and Arendt $\mathrm{T}$. The significance of the cholinergic system in the brain during aging and in Alzheimer's disease. J Neural Transm 2006; ; 113: 1625-1644

[47] Jann MW, et al. Clinical pharmacodynamics of cholinesterase inhibitors. Clin Pharmacokinet 2004; 41: 719-739

[48] Li J, et al. Huperzine A for Alzheimer's disease. Cochrane Database Syst Rev 2008; 16: CD005592

[49] Rafii MS, et al. A phase II trial of huperzine A in mild and moderate Alzheimer disease. Neurology 2011; 76: 1389-1394

[50] Raina $\mathrm{P}$, et al. Effectiveness of cholinesterase inhibitors and memantine for treating dementia: evidence review for a clinical guideline. Ann Intern Med 2008; 148: 379-397

[51] Hogan D, et al. Comparison studies of cholinesterase inhibitors for Alzheimer's disease. Lancet Neurol 2004; 3: 622-626

[52] Cacabellos R, et al. Pharmacogenetic aspects of therapy with cholinesterase inhibitors: the role of CYP2D6 in Alzheimer's disease pharmacogenetics. Curr Alzheimer Res 2007; 4: 479-500

[53] Giacobini E. Selective inhibitors of butyrylcholinesterase: a valid alternative for therapy of Alzheimer's disease? Drugs Aging 2001; 18: $891-898$

[54] Lane RM, et al. Targeting acetylcholinesterase and butyrylcholinesterase in dementia. Int $\mathrm{J}$ Neuropsychopharmacol 2006; 9: 101-124

[55] Weinstock M. Selectivity of cholinesterase inhibition: clinical implications for the treatment of Alzheimer's disease. CNS Drugs 1999; 12: 307-323

[56] Richardson C et al. Blood pro-inflammatory cytokines in Alzheimer's disease in relation to the use of acetylcholinesterase inhibitors. Int J Geriatr Psychiatry 2013; 28: 1312-1317

[57] Rogers SL, et al. Pharmacokinetic and pharmacodynamic profile of donepezil HCI following multiple oral doses. Br J Clin Pharmacol 1998; 46(Suppl 1): 7-12

[58] Tiseo PJ, et al. Metabolism and elimination of 14C-donepezil in healthy volunteers: a single dose study. Br J Clin Pharmacol 1998; 46(Suppl 1): 19-24

[59] Ohnishi A, et al. Comparison of the pharmacokinetics of E2020, a new compound for Alzheimer's disease, in healthy young and elderly subjects. J Clin Pharmacol 1993; 33: 1086-1091

[60] Hansen R, et al. Efficacy and safety of donepezil, galantamine, and rivastigmine for the treatment of Alzheimer's disease: a systematic review and meta-analysis. Clin Interv Aging 2008; 3: 211-225.

[61] Birks J and Harvey R. Donepezil for dementia due to Alzheimer's disease. Cochrane Database Syst Rev 2006; 25: CD001190.

[62] Ritchie C. et al. Metanalysis of randomized trials of the efficacy and safety of donepezil, galantamine and rivastigmine for the treatment of Alzheimer's disease. Am J Geriatr Psychiatry 2004; 12: 358-369.

[63] Holmes C, et al. The efficacy of donepezil in the treatment of neuropsychiatric symptoms in Alzheimer's disease. Neurology 2004; 363: 2100-2101

[64] Howard R, et al. Donepezil for the treatment of agitation in Alzheimer's disease. N Engl J Med 2007; 357: 1382-1392

[65] Farlow MR, et al. Effectiveness and tolerability of high-dose (23 $\mathrm{mg} / \mathrm{d})$ versus standard-dose $(10 \mathrm{mg} / \mathrm{d})$ donepezil in moderate to severe Alzheimer's disease: a 24-week, randomized, double-blin study. Clin Ther 2010; 32: 1234-1251.
[66] Hwang $\mathrm{J}$ et al. Microglia signaling as a target of donepezil. Neuropharmacology 2010: 58: 1122-1129

[67] Kim HG et al. Donepezil inhibits the amyloid-beta oligomerinduced microglial activation in vitro and in vivo. Neurotoxicology 2014; 40: 23-32

[68] Meunier J et al. Brain toxicity and inflammation induced in vivo in mica by the amyloid- $\beta$ forty-two inducer aftin- 4 , a roscovitine derivative. J Alzheimers Dis 2015; 44: 507-524.

[69] Tyagi E et al. Effect of anti-dementia drugs on LPS induced neuroinflammation in mice. Life Sci 2007; 80: 1977-1983

[70] Yoshiyama $\mathrm{Y}$ et al. Anti-inflammatory action of donepezil ameliorates tau pathology, synaptic loss and neurodegeneration in a tauopathy mouse model. J Alzheimers Dis 2010; 22: 295-306.

[71] Reale $\mathrm{M}$ et al. The acetylcholinesterase inhibitor, Donepezil, regulates a Th2 bias in Alzheimer's disease patients. Neuropharmacology 2006; 50: 606-613

[72] Gambi F et al. Alzheimer patients treated with an AchE inhibitor show higher IL-4 and lower IL-1 beta levels and expression in peripheral blood mononuclear cells. J Clin Psychopharmacol 2004; 24: 314-321

[73] Lilienfeld S. Galantamine-A novel cholinergic drug with a unique dual mode of action for the treatment of patients with Alzheimer's disease. CNS Drug Rev 2002; 8: 159-176

[74] Zhao Q, et al. Pharmacokinetics of extended-release and immediate-release formulations of galantamine at steady state in healthy volunteers. Curr Med Res Opin 2005; 21: 1547-1554

[75] Seltzer B. Galantamine-ER for the treatment of mild to moderate Alzheimer's disease. Clin Interv Aging 2010; 5: 1-6

[76] Mannens GS et al. The metabolism and excretion of galantamine in rats, dogs and humans. Drug Metab Dispos 2002; 30: 553-563

[77] Piotrovsky V et al. Galantamine population pharmacokinetics in patients with Alzheimer's disease: modeling and simulations. J Clin Pharmacol 2003; 43: 514-523

[78] Zhao Q et al. Pharmacokinetics and safety of galantamine in subjects with hepatic impairment and healthy volunteers. J Clin Pharmacol 2002; 42: 428-436

[79] 79- Pavlov VA et al. The anti-inflammatory efficacy of galantamine is dependent on the integrity of the cholinergic antiinflammatory pathway. Shock 2007; 27: 23

[80] Pavlov VA et al. Brain acetylcholinesterase activity controls systemic cytokine levels through the cholinergic anti-inflammatory pathway. Brain Behav and Immun 2009; 23: 41-45

[81] Liu ZH et al. Effect of cholinesterase inhibitor galanthamine on circulating tumor necrosis factor alpha in rats with lipopolysaccharide-induced peritonitis. Chin Med J 2010; 123: 1727-1730

[82] Satapathy SK et al. Galantamine alleviates inflammation and other obesity-associated complications in high-fat diet-fed mice. Mol Med 2011; 17: 599-606.

[83] Giunta B et al. Galantamine and nicotine have a synergistic effect on inhiibbition of microglial activation induced by HIV-1 gp1200. Brain Res Bull 2004; 64: 165-170.

[84] Takata $\mathrm{K}$ et al. Galantamine-induced Amyloid- $\beta$ clearance mediated via stimulation of microglial nicotinic acetylcholine receptors. J Biol Chem 2010; 285: 40180-40191.

[85] Polinsky R. Clinical pharmacology of rivastigmine: a new generation acetylcholinesterase inhibitor for the treatment of Alzheimer's disease. Clin Ther 1998; 20: 634-647.

[86] Hossain M, et al. Estimation of the absolute bioavailability of rivastigmine in patients with mild to moderate dementia of the Alzheimer's type. Clin Pharmacokinet 2002; 41: 225-234.

[87] Lefevre G, et al. Pharmacokinetics and pharmacodynamics of the novel daily rivastigmine transdermal patch compared with twicedaily capsules in Alzheimer's disease patients. Clin Pharmacol Ther 2008; 83: 106-114.

[88] Cutler NR, et al. Dose-dependent CSF acetylcholinesterase inhibition by SDZ ENA 713 in Alzheimer's disease. Acta Neurol Scand 1998; 97: 244-250.

[89] Birks J, et al. Rivastigmine for Alzheimer's disease. Cochrane Database Syst Rev 2009; 2: CD001191.

[90] Winbland B, et al. IDEAL: a 6-month, double-blind, placebocontrolled, study of the first skin patch for Alzheimer disease. Neurology 2007; 69(suppl 1): S14-22.

[91] Nazri E et al. Suppression of neuroinflammation and immunomodulation by the acetylcholinesterase inhibitor rivastigmine. J Neuroimmunol 2008; 203: 12-22. 
[92] Shifrin $\mathrm{H}$ et al. Rivastigmine alleviates experimentally induced colitis in mice and rats by acting at central and peripheral sites to modulate immune responses. PLoS One 2013; 8: e57668.
[93] Gubandru M et al. Alzheimer's disease treated patients showed different patterns for oxidative stress and inflammation markers. Food Chem Toxicol 2013; 61: 209-214. 